\title{
AREA OF BINOCULAR VISION IN ANOMALOUS RETINAL CORRESPONDENCE*
}

\author{
BY \\ L. PASINO AND G. MARAINI \\ From the Ophthalmological Clinic of the Parma Medical School, Italy: Director, Professor P. Matteucci
}

SEVERAL authors (Lyle and Foley, 1955; Naylor, Shannon, and Stanworth, 1956; Naylor and Stanworth, 1959; Guzzinati and Salvi, 1957; Pasino, Maraini, and Santori, 1963) have observed the absence of a stereoscopic perception of depth in early convergent strabismus with anomalous retinal correspondence. Even in patients with an extremely small fixed ocular deviation and harmonious anomalous retinal correspondence (h.a.r.c.), the stereoscopic visual acuity is not significantly different from that of subjects with deep uniocular amblyopia (Pasino and others, 1963).

The interpretation of these observations is by no means certain, considering that the sensory condition of these patients permitted a subnormal biretinal cooperation with a certain range of fusional amplitude; the latter was shown to be almost exclusively based on variations of the angle of anomaly rather than on true fusional movements (Halldén, 1952; Pasino and Maraini, 1964; Maraini and Pasino, 1964).

Comparing sensory fusion in normal and anomalous binocular vision, Halldén (1952) pointed out that sensory fusion in anomalous correspondence is neither confined within such narrow limits nor characterized by the occurrence of stereo-effects. According to Halldén (1952), a large amplitude of sensory fusion could not be expected to occur without a loss of stereopsis.

In the present investigation an attempt has been made to evaluate the amplitude of sensory fusion and therefore the range of binocular vision in patients with fixed small-angle early convergent strabismus with h.a.r.c. and in a few patients of this kind in whom orthophoria had been attained (by means of surgery and of prisms) together with a normal binocular localization in free space. As far as we know this subject has not been discussed in the literature.

\section{Material and Methods}

The methods used to calculate the transverse magnitude of the area of binocular vision was a modification of the apparatus described by Ogle (1962). These modifications were indispensable in order to make sure that the patient retained h.a.r.c. throughout the testing and that important suppressive phenomena did not occur.

The patient's head is firmly held by a head-rest and the subject steadily fixes a luminous fixation point at the top of a vertical rod placed on the median plane at a distance of one metre. A similar vertical black rod, having at its top a luminous point of a different colour, is mounted on a carriage

* Received for publication June 18, 1965. 
that can be moved along a fixed track, the proximal end of which lies directly below the pupil of the left eye. As this rod is moved along the track nearer to and further from the fixation point, the distal and proximal positions in which the luminous point appears double can usually be determined; with the aid of a pair of Bagolini's striated glasses placed in front of the patient the examiner can check that the subject is retaining h.a.r.c. during the testing and that no suppression of one of the two half-images of the moving light oceurs. If the experimental determination of the distal and proximal limits of the region of binocular single vision is carried out for a number of lateral angles from the fixation point, a spatial representation of Panum's area can be plotted.

With this technique we examined eleven patients ranging in age from 8 to 32 years. Seven had a small-angle convergent squint with h.a.r.c.; the ocular deviation was less than 6 prism dioptres and the visual acuity of the deviating eye was at least $4 / 10$. Four patients had an early convergent strabismus in which orthophoria had been obtained (with the aid of surgery and of prisms) for a period ranging from 1 month to 2 years. At the time of testing all these patients had normal binocular localization when examined in normal surroundings with the striated glasses; two of them still retained anomalous retinal correspondence when tested on the synoptophore or with Hering-Bielschowsky after-images. None of them had a stereoscopic visual acuity within the normal range.

The "good cooperation" required of the patient and the subjective difficulties of the testing procedure justify, we think, the relatively small number of subjects examined. All of them have been tested several times on different days until constant answers were obtained.

\section{Results}

The first diagram (overleaf) illustrates the spatial representation of the mean values of the limits of the region of binocular single vision in normal subjects in the same age range when tested under the same experimental conditions.

The diagrams numbered 1 to 7 (overleaf) summarize the results obtained in seven cases of small-angle convergent strabismus with h.a.r.c. In three of them (Nos $2,5,7)$ the posterior limits of the region of binocular vision could not be determined because of the impossibility of eliciting diplopia distal to the fixation point; in these same cases the proximal limit of the area of binocular vision is significantly shifted nearer to the patient if compared with normal subjects. The remaining four cases of small-angle strabismus (Nos $1,3,4,6$ ) showed a constant widening of the area of binocular vision, more evident when the distal limit is considered.

Essentially similar results were obtained in four patients (diagrams numbered 8 to 11 , overleaf) in whom orthophoria with normal binocular localization had been reached with the aid of surgery and correction with prisms; again, the area of binocular vision was found to be widened if compared with that detected in normal subjects.

\section{Discussion}

As was suggested by Halldén (1952) and could be anticipated by previous work on the easy variability of the angle of anomaly in anomalous correspondence, the area of sensory fusion seems to be significantly enlarged in small-angle convergent strabismus with h.a.r.c. This large amplitude of sensory fusion might reasonably be interpreted as a sufficient cause of the absence of stereoscopic visual acuity in this condition. This seems to be the case even in the presence of a recently-gained orthophoria with normal binocular localization in free space. In effect, previous observations by Maraini and Pasino (1965) would seem to indicate that, in early convergent strabismus, the normal binocular localization which is immediately present after orthophoria is obtained, does not possess the characteristics of a stable 


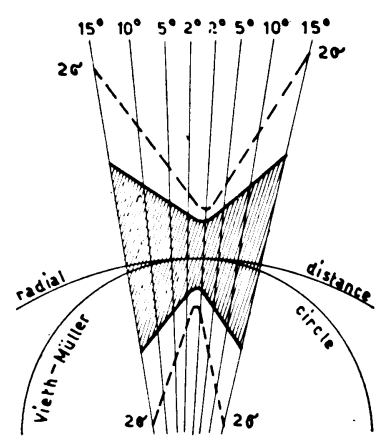

NORMAL

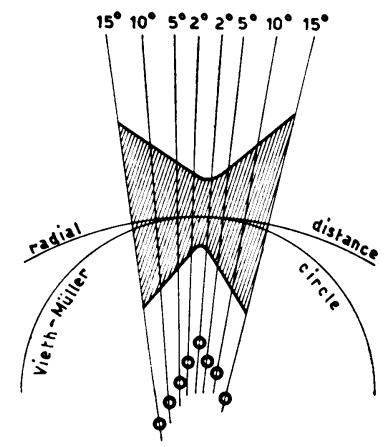

2

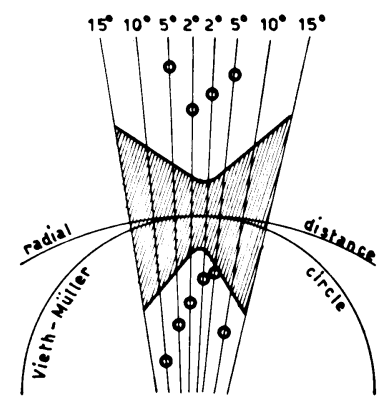

4

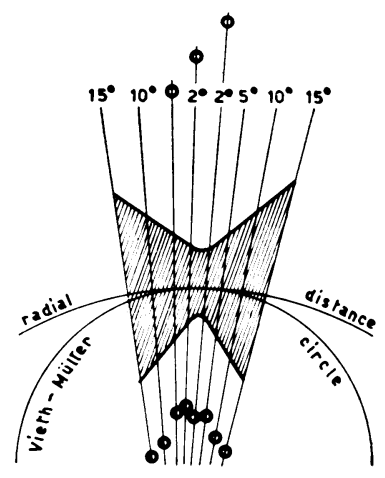

I

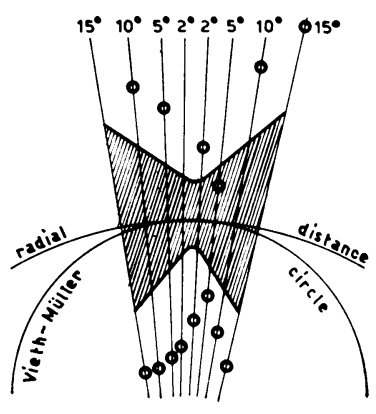

3

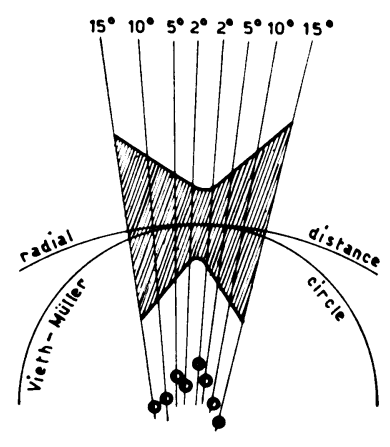

5

Normal: Mean values of the limits of the region of binocular single vision in normal subjects with double standard deviation.

CASES 1 to 7: Spatial representation of the region of binocular vision in seven cases of small angle convergent squint with harmonious anomalous correspondence. 


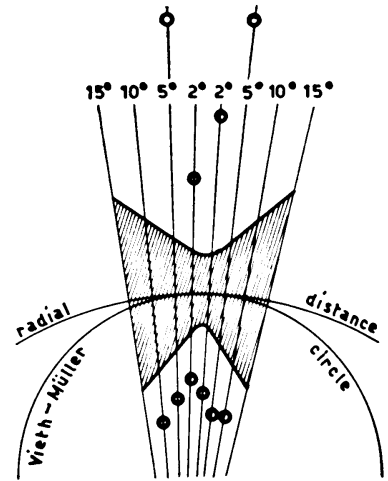

6

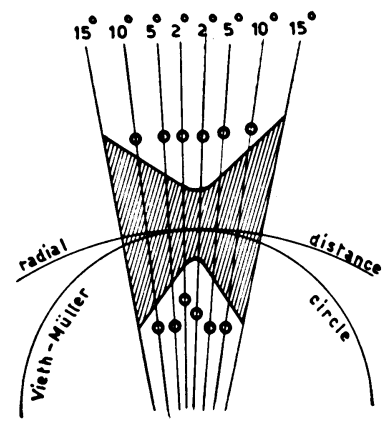

8

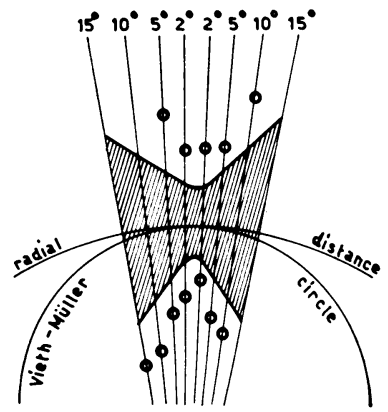

10

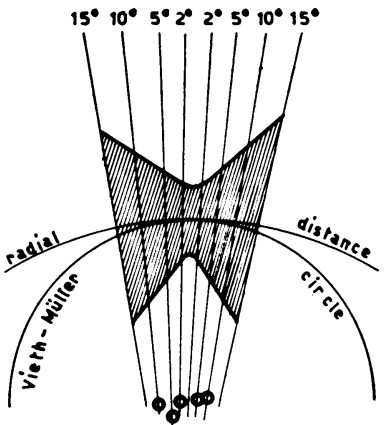

7

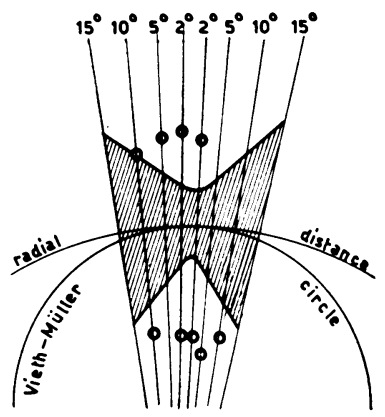

9

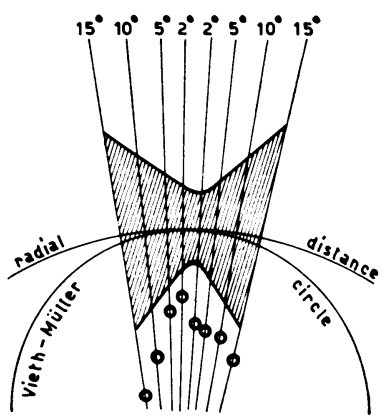

11

CASES 8 to 11: Spatial representation of the region of binocular vision in four patients with orthophoria (following surgery or correction with prisms) and normal binocular localization in normal surroundings. 
functional relationship peculiar to a fully developed retinal correspondence. In normal surroundings, a certain fusional amplitude is obtained by means of variations of the angle of anomaly and stereoscopic acuity is absent. Stereopsis could be gained in some cases only if orthophoria is maintained over a sufficient lapse of time and if the patient's binocular vision is still in a plastic stage.

The opportunity to determine the possible modification of the area of sensory fusion in a sufficient number of these patients during a certain length of time could further support this hypothesis.

\section{Summary}

The amplitude of the area of sensory fusion has been determined in patients with small-angle strabismus with harmonious anomalous retinal correspondence and in a small number of patients of the same type who attained orthophoria with normal binocular localization in free space. A constant widening of the area of binocular vision was detected in these patients when compared to normal subjects.

The possible significance of these findings is briefly discussed in view of the absence of stereoscopic visual acuity in these patients.

\section{REFERENCES}

Guzzinati, G. C., and Salvi, G. (1957). Ann. Ottal., 83, 417.

Halldén, U. (1952). Acta ophthal. (Kbh.), Suppl. 37.

LyLe, T. Keith, and Foley, J. (1955). Brit. J. Ophthal., 39, 474.

Maraini, G., and Pasino, L. (1964). Ibid., 48, 439.

- - (1965). Ibid., 49, 154.

Naylor, E. J., Shannon, T. E., and Stanworth, A. (1956). I Ibid., 40, 641. and Stanworth, A. (1959). Ibid., 43, 662.

Ogle, K. N. (1962). In "The Eye", ed. H. Davson, vol. 4, p. 276. Academic Press, New York and London.

Pasino, L., and Maraini, G. (1964). Brit. J. Ophthal., 48, 30.

$\longrightarrow$, , and SANTORI, M. (1963). Ann. Ottal., 89, 1005.

\section{ADDENDUM}

When this paper was ready for publication a paper on the same subject by B. Bagolini and $\mathrm{N}$. Capobianco appeared in Amer. J. Ophthal. (1965), 59, 430. We are glad to see the perfect agreement of their results with our findings. 